\title{
Critical Temperature of Heisenberg Models on Regular Trees, via Random Loops
}

\author{
Jakob E. Björnberg ${ }^{1} \cdot$ Daniel Ueltschi ${ }^{2}$
}

Received: 30 March 2018 / Accepted: 7 September 2018 / Published online: 25 September 2018

(c) The Author(s) 2018

\section{Abstract}

We estimate the critical temperature of a family of quantum spin systems on regular trees of large degree. The systems include the spin- $\frac{1}{2} \mathrm{XXZ}$ model and the spin- 1 nematic model. Our formula is conjectured to be valid for large-dimensional cubic lattices. Our method of proof uses a probabilistic representation in terms of random loops.

Keywords Random loop model · Quantum Heisenberg · Critical temperature

Mathematics Subject Classification $60 \mathrm{~K} 35 \cdot 82 \mathrm{~B} 20 \cdot 82 \mathrm{~B} 26 \cdot 82 \mathrm{~B} 31$

\section{Introduction and Main Result}

The main goal of this study is to predict an expression for the critical temperature of a family of quantum spin systems on the cubic lattice $\mathbb{Z}^{v}$ that holds asymptotically for large dimension $v$. More precisely, we propose the first two terms in the expansion in powers of $v^{-1}$. The family of quantum spin systems includes the spin $\frac{1}{2}$ ferromagnetic and antiferromagnetic Heisenberg models and the XXZ model. We also consider spin 1 quantum nematic systems. Our results are expected to be exact but they are not rigorous on $\mathbb{Z}^{v}$. In fact we do not perform calculations with the cubic lattice but we consider the model on regular trees with $d$ descendants; we obtain the first two terms of the critical inverse temperature in powers of $d^{-1}$. For trees our computations are completely rigorous. We conjecture that our expression applies to $\mathbb{Z}^{v}$ when taking $d=2 v-1$.

Jakob E. Björnberg

jakob.bjornberg@gu.se

Daniel Ueltschi

daniel@ueltschi.org

1 Department of Mathematical Sciences, Chalmers University of Technology and the University of Gothenburg, Gothenburg, Sweden

2 Department of Mathematics, University of Warwick, Coventry CV4 7AL, United Kingdom 


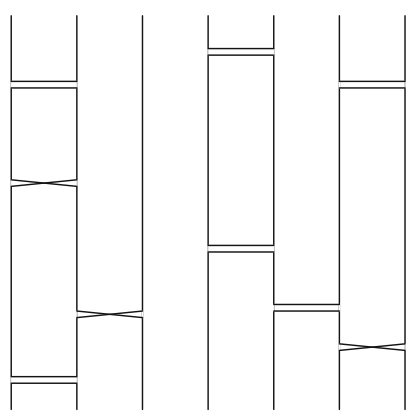

Fig. 1 Random loops coming from a configuration $\omega$ of crosses and bars, in the case when the underlying graph is a line with seven vertices. To each vertex corresponds a vertical line segment which is a copy of the interval $[0,1]$. On following a loop one reverses direction when traversing a double-bar, maintains direction when traversing a cross, and proceeds periodically in the vertical direction. In this example there are $\ell(\omega)=4$ loops

\subsection{Random-Loop Model}

Our method is based on using a random loop representation, which we now describe. The relevant model of random loops may be defined for arbitrary finite graphs, here we consider mainly trees. Let $T$ denote an infinite rooted tree where each vertex has $d \geq 2$ offspring, and write $\rho$ for its root. We sometimes refer to the number of offspring of vertex as its outdegree. For $m \geq 0$ let $T_{m}$ denote the subtree of $T$ consisting of the first $m$ generations ( $\rho$ being generation zero). Write $V_{m}$ and $E_{m}$ for the vertex- and edge sets of $T_{m}$.

Let $\mathbb{P}_{m}(\cdot)$ denote a probability measure governing a collection $\omega=\left(\omega_{x y}: x y \in E_{m}\right)$ of independent Poisson processes on the interval $[0,1]$, indexed by the edge-set $E_{m}$, each having rate $\beta$ (the inverse-temperature). We refer to realizations of $\omega$ as a collections of links, and to $\omega_{x y}$ as the links supported by the edge $x y$. Thus, disjoint sub-intervals $I, J \subseteq[0,1]$ independently receive uniformly placed links, their number being Poisson-distributed with mean $\beta|I|$ and $\beta|J|$, respectively. We write $\mathbb{E}_{m}[\cdot]$ for expectation under $\mathbb{P}_{m}(\cdot)$.

A given link is assigned to be a cross with probability $u$, otherwise a double-bar, independently between different links. The collection of links then decomposes $T_{m} \times[0,1]$ into a collection of disjoint loops in a natural way. Rather than giving a formal definition here, we refer to Fig. 1. A formal definition may be found e.g. in [17, Sect. 2.1].

The total number of loops is denoted $\ell=\ell(\omega)$. We actually work with a weighted version of $\mathbb{P}_{m}(\cdot)$, denoted $\mathbb{P}_{m}^{(\theta)}(\cdot)$ with a positive parameter $\theta$. This is the probability measure whose expectation operator $\mathbb{E}_{m}^{(\theta)}[\cdot]$ is given by

$$
\mathbb{E}_{m}^{(\theta)}[X]=\frac{\mathbb{E}_{m}\left[X \theta^{\ell(\omega)}\right]}{\mathbb{E}_{m}\left[\theta^{\ell(\omega)}\right]}
$$

Note that $\mathbb{P}_{m}^{(1)}=\mathbb{P}_{m}$.

All loops are small when $\beta$ is small, this may be shown e.g. as in [9, Thm. 6.1]. But it is expected that there exists $\beta_{\mathrm{c}}$, that depends on the parameter $\theta$ and the outdegree $d$, such that a given points lies in an infinite loop with positive probability for $\beta>\beta_{\mathrm{c}}$. Our main result is a formula for $\beta_{\mathrm{c}}$; it is asymptotic in the outdegree $d \rightarrow \infty$, namely

$$
\frac{\beta_{\mathrm{c}}}{\theta}=\frac{1}{d}+\frac{1-\theta u(1-u)-\frac{1}{6} \theta^{2}(1-u)^{2}}{d^{2}}+o\left(d^{-2}\right),
$$


and we can prove that there are infinite loops for $\beta>\beta_{\mathrm{c}}$ in the vicinity of $\beta_{\mathrm{c}}$. For a more precise statement, see Theorem 1.1 below.

The first study of this model on trees is due to Angel [2], who established the presence of long loops for a range of parameters $\beta$ when $d \geq 4$; he only considered the case $u=1$ and $\theta=1$. Angel's results were extended by Hammond [10,11]; he gave a precise characterisation of the critical parameter $\beta_{\mathrm{c}}$ for large enough $d$. The Formula (1.1) was established in [6] in the case $\theta=1$, our study following a suggestion of Hammond. Very recently Hammond and Hegde [12] proved that the Formula (1.1) for $\theta=1$ truly identifies the critical point, not only in the local sense considered here and in [6]; their results hold for large $d$. Another extension to $\theta \neq 1$ has independently been proposed by Betz et al. [4].

\subsection{Quantum Spin Systems}

Let $(\Lambda, \mathcal{E})$ denote a graph, with $\Lambda$ the set of vertices and $\mathcal{E}$ the set of edges. The main examples to bear in mind here are regular trees, and finite subsets of $\mathbb{Z}^{d}$ with nearest-neighbour edges. The spin- $\frac{1}{2}$ systems have Hilbert space $\mathcal{H}_{\Lambda}=\otimes_{x \in \Lambda} \mathbb{C}^{2}$ and the hamiltonian is

$$
H_{\Lambda}=-2 \sum_{\{x, y\} \in \mathcal{E}}\left(S_{x}^{(1)} S_{y}^{(1)}+S_{x}^{(2)} S_{y}^{(2)}+\Delta S_{x}^{(3)} S_{y}^{(3)}\right),
$$

where $S_{x}^{(i)}, i=1,2,3$ denotes the $i$ th spin operator at site $x \in \Lambda$. Here, $\Delta \in[-1,1]$ is a parameter.

As was progressively understood in $[1,15,17]$, this quantum system is represented by the model of random loops with $\theta=2$ and $u=\frac{1}{2}(1+\Delta)$. Indeed, the quantum two-point correlation function is given by loop correlations,

$$
\left\langle S_{x}^{(1)} S_{y}^{(1)}\right\rangle:=\frac{\operatorname{tr}\left(S_{x}^{(1)} S_{y}^{(1)} \mathrm{e}^{-\beta H_{\Lambda}}\right)}{\operatorname{tr}\left(\mathrm{e}^{-\beta H_{\Lambda}}\right)}=\frac{1}{4} \mathbb{P}_{\Lambda}^{(\theta=2)}(x \leftrightarrow y),
$$

where $\{x \leftrightarrow y\}$ is the event that $(x, 0)$ and $(y, 0)$ belong to the same loop. It follows that magnetic long-range order is related to the occurrence of large loops.

On $\mathbb{Z}^{3}$, the critical inverse temperature has been computed numerically; it was found that

$$
\beta_{\mathrm{c}}^{(\nu=3)}(\Delta)= \begin{cases}0.596 \ldots & \text { if } \Delta=1 ; \text { Troyer et al. [16] } \\ 0.4960 \ldots & \text { if } \Delta=0 ; \text { Wessel, private communication in [3] } \\ 0.530 \ldots & \text { if } \Delta=-1 ; \text { Sandvik [13], Troyer et al. [16] }\end{cases}
$$

For large $v$, the lattice $\mathbb{Z}^{v}$ behaves like a tree of outdegree $d=2 v-1$. Our Formula (1.1) gives

$$
\beta_{\mathrm{c}}^{(v)}(\Delta)=\frac{1}{v}+\frac{1}{v^{2}}\left[1-\frac{1}{6}(1-\Delta)(2+\Delta)\right]+o\left(\frac{1}{v^{2}}\right) .
$$

With $v=3$, the values for $\Delta=1,0,-1$ are $\frac{4}{9}, \frac{11}{27}, \frac{11}{27}$ respectively. They corroborate the numerical values (1.3) to some extent. Of course, the Formula (1.4) gets more accurate in high dimensions.

In the case of spin-1 systems, the Hilbert space is $\mathcal{H}_{\Lambda}=\otimes_{x \in \Lambda} \mathbb{C}^{3}$ and the hamiltonian is

$$
H_{\Lambda}=-\sum_{\{x, y\} \in \mathcal{E}}\left(u \vec{S}_{x} \cdot S_{y}+\left(\vec{S}_{x} \cdot S_{y}\right)^{2}\right),
$$

See [17]. The phase diagram of this model was determined in [7]. For $0<u<1$ the system displays nematic long-range order at low temperatures (if $d \geq 3$; also in the ground 
state when $d=2)$. This was rigorously proved in [14,17]. The corresponding loop model has parameter $\theta=3$, and the same $u$ as in (1.5). Loop correlations are related to nematic long-range order, namely

$$
\left\langle A_{x} A_{y}\right\rangle=\frac{2}{9} \mathbb{P}_{\Lambda}^{(\theta=3)}(x \leftrightarrow y),
$$

with $A_{x}=\left(S_{x}^{(3)}\right)^{2}-\frac{2}{3}$. We are not aware of numerical calculations of the critical inverse temperature $\beta_{\mathrm{c}}$ for this model on $\mathbb{Z}^{3}$. With $\theta=3$ and $d=2 v-1$, the Formula (1.1) gives

$$
\beta_{\mathrm{c}}^{(v)}(u)=\frac{3}{2 v}+\frac{3}{2 v^{2}}\left[1-\frac{3}{4}\left(1-u^{2}\right)\right]+o\left(\frac{1}{v^{2}}\right) .
$$

\subsection{Main Result}

In the rest of this paper we deal only with the probabilistic model of random loops defined above, and we allow $\theta$ to be any (fixed) positive real number. Our main result is that, as the distance between $x$ and $y$ goes to $\infty$, the two-point function vanishes or stays positive, according to whether $\beta$ is smaller or larger than $\beta_{\mathrm{c}}$ given above. Let us say that a loop visits a vertex $x$ of $T_{m}$ if the loop contains a point $(x, t)$ for some $t \in[0,1]$. Motivated by (1.2) and (1.6) we consider

$$
\sigma_{m}=\mathbb{P}_{m}^{(\theta)}(\rho \leftrightarrow m),
$$

that is, $\sigma_{m}$ is the $\mathbb{P}_{m}^{(\theta)}$-probability that $(\rho, 0)$ belongs to a loop which visits some vertex in generation $m$ in $T_{m}$.

Throughout this paper we work with $\beta$ of the form

$$
\frac{\beta}{\theta}=\frac{1}{d}+\frac{\alpha}{d^{2}}, \quad \text { where }|\alpha| \leq \alpha_{0}
$$

for some fixed but arbitrary $\alpha_{0}>0$. All error terms $O(\cdot), o(\cdot)$ and constants may depend on $\alpha_{0}$ but are otherwise uniform in $\alpha$.

Theorem 1.1 Consider $\beta$ of the form (1.7), and write

$$
\alpha_{*}=\alpha_{*}(\theta, u)=1-\theta u(1-u)-\frac{1}{6} \theta^{2}(1-u)^{2} .
$$

For any $\delta>0$ there exists $d_{0}=d_{0}\left(\theta, u, \alpha_{0}, \delta\right)$ such that for $d \geq d_{0}$ we have:

- if $\alpha \leq \alpha_{*}-\delta$ then $\lim _{m \rightarrow \infty} \sigma_{m}=0$;

- if $\alpha \geq \alpha_{*}+\delta$ then $\liminf \inf _{m \rightarrow \infty} \sigma_{m}>0$.

Let us remark that for $\theta=1$ the result was shown in our previous work [6]. The arguments presented here are strengthened versions of those arguments. The basic strategy is to establish recursion inequalities for the sequence $\sigma_{m}$, see Proposition 2.1. These are obtained by analyzing the local configuration around the root $\rho$, in particular we identify two events $A_{1}$ and $A_{2}$ which together contribute most of the probability in the regime we consider $(d \rightarrow \infty$ and $\beta$ as in (1.7)). 


\section{Proof of the Main Result}

The indicator function of an event $A$ will be written $\mathbb{I}_{A}$ or $\mathbb{I}\{A\}$. The partition function for the loop model on $T_{m}$ is written $Z_{m}=\mathbb{E}_{m}\left[\theta^{\ell}\right]$. For convenience we also define

$$
z_{m}=e^{-d \beta(1-1 / \theta)} \frac{\theta Z_{m-1}^{d}}{Z_{m}} .
$$

For given $m \geq 1$ and $\varepsilon>0$ we define

$$
\tilde{\sigma}_{m}=\sigma_{m} \wedge \sigma_{m-1} \wedge\left(\frac{\varepsilon}{d}\right) .
$$

(A priori we need not have $\sigma_{m} \leq \sigma_{m-1}$ since they are computed using different measures.) In this section we will prove the following recursion-inequalities.

Proposition 2.1 For all $m \geq 1$ we have

$$
\sigma_{m} \geq \tilde{\sigma}_{m-1}+\frac{\tilde{\sigma}_{m-1}}{d}\left(\alpha-\alpha_{*}\right)-\frac{1}{2} \tilde{\sigma}_{m-1}^{2}+O\left(d^{-3}\right),
$$

and

$$
\sigma_{m} \leq\left(\sigma_{m-1} \vee \sigma_{m-2}\right)\left[1+\frac{1}{d}\left(\alpha-\alpha_{*}\right)+O\left(d^{-2}\right)\right]
$$

Here the $O\left(d^{-3}\right)$ and $O\left(d^{-2}\right)$ are uniform in $m$.

Our main result follows easily:

Proof of Theorem 1.1 First suppose $\alpha<\alpha_{*}$. For $d$ large enough the factor in square brackets in (2.3) is strictly smaller than 1 . This easily gives that $\sigma_{m}$ decays to 0 exponentially fast.

Now suppose $\alpha>\alpha_{*}$. Clearly $\sigma_{0}=1$, and it is not hard to see that there exists a constant $c_{1}>0$ such that $\sigma_{1} \geq c_{1}$ for all $d$. This implies that $\tilde{\sigma}_{1}=\varepsilon / d$ if $\varepsilon<c_{1}$. If also $\varepsilon<2\left(\alpha-\alpha_{*}\right)$ and $d$ is large enough then (2.2) and induction on $m$ give that $\sigma_{m} \geq \tilde{\sigma}_{m}=\varepsilon / d$ for all $m \geq 1$.

Before turning to the proof of Proposition 2.1, let us describe some of the main ideas and also what new input is required compared to our previous work [6] on the case $\theta=1$. For the lower bound (2.2) we will estimate the probability of certain local configurations near $\rho$ which guarantee that $\rho$ is connected to generation $m$ if certain of its children (or grandchildren) are. For the upper bound we similarly estimate $\mathbb{P}_{m}^{(\theta)}(\rho \nLeftarrow m)$ in terms of the probability that certain of $\rho$ 's children (or grandchildren) are blocked from generation $m$. When $\theta \neq 1$, the configurations in the subtrees rooted at the children of $\rho$ are not independent of the local configuration adjacent to $\rho$. Thus we must deal carefully with the factor $\theta^{\ell(\omega)}$ and how it behaves in the local configurations which we consider. This involves obtaining estimates for the partition function $Z_{m}$ in terms of the partition function $Z_{m-1}$ in the smaller tree, which is where the number $z_{m}$ in (2.1) becomes relevant.

As was the case in [6], the hardest part is the upper bound (2.3). This is because we must rule out connections due to 'lower order events' $\left(\left(A_{1} \cup A_{2}\right)^{c}\right.$ in the notation below) where the loop structure is too complicated to handle directly. The main technical advance compared to [6] started with a simplification of the argument used there to deal with this difficulty. Having this simpler version allowed us to deal also with the correlations caused by the factor $\theta^{\ell(\omega)}$, see Proposition 2.5. 

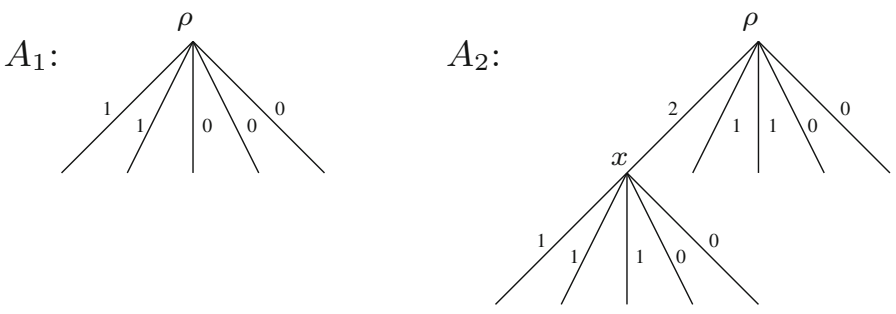

Fig. 2 Illustrations of the two events $A_{1}$ and $A_{2}$. Numbers on edges indicate the number of links

\subsection{Preliminary Calculations}

Let us first introduce some notations and prove some facts that will be used for establishing both bounds in Proposition 2.1.

Write $A_{1}$ for the event that, for each child $x$ of $\rho$, there is at most one link between $\rho$ and $x$. Write $A_{2}$ for the event that: (i) there is a unique child $x$ of $\rho$ with exactly 2 links between $\rho$ and $x$, (ii) for all siblings $x^{\prime}$ of $x$ there is at most one link between $\rho$ and $x^{\prime}$, and (iii) for all children $y$ of $x$ there is at most one link between $x$ and $y$. See Fig. 2.

Let $\zeta_{m}=1-\sigma_{m}$ and let $B_{m}^{\rho}$ be the event that $(\rho, 0)$ does not belong to a loop which reaches generation $m$ in $T_{m}$, thus $\mathbb{P}_{m}^{(\theta)}\left(B_{m}^{\rho}\right)=\zeta_{m}$. Clearly we have that

$$
\zeta_{m}=\mathbb{P}_{m}^{(\theta)}\left(B_{m}^{\rho}\right)=\mathbb{P}_{m}^{(\theta)}\left(B_{m}^{\rho} \cap A_{1}\right)+\mathbb{P}_{m}^{(\theta)}\left(B_{m}^{\rho} \cap A_{2}\right)+\mathbb{P}_{m}^{(\theta)}\left(B_{m}^{\rho} \backslash\left(A_{1} \cup A_{2}\right)\right)
$$

Let us enumerate the children of $\rho$ by $i=1, \ldots, d$ and let $\ell_{i}$ denote the number of loops in the restriction of $\omega$ to the subtree to distance $m$ rooted at child $i$. On the event $A_{1}$, and if there are $k$ links from $\rho$, the number $\ell$ of loops satisfies

$$
\ell=\sum_{i=1}^{d} \ell_{i}-k+1
$$

To see this, one may imagine that the $k$ links to $\rho$ are put in last, one at a time. Each such link then merges some loop in the corresponding subtree with a loop visiting $\rho$. (This uses the tree-structure of the underlying graph, which implies that there can be no connections between $\rho$ and the subtree until the link is put in.) It follows that

$$
\begin{aligned}
\mathbb{E}_{m}\left[\theta^{\ell} \mathbb{I}_{A_{1}}\right] & =\sum_{k=0}^{d} \theta^{-k+1} \mathbb{E}_{m}\left[\theta^{\sum_{i} \ell_{i}} \mathbb{I}_{A_{1}} \mathbb{I}\{k \text { links at } \rho\}\right] \\
& =\theta \sum_{k=0}^{d}\left(\begin{array}{l}
d \\
k
\end{array}\right)\left(e^{-\beta}\right)^{d-k}\left(e^{-\beta} \frac{\beta}{\theta}\right)^{k} Z_{m-1}^{d} \\
& =\theta Z_{m-1}^{d}\left(e^{-\beta}\left(1+\frac{\beta}{\theta}\right)\right)^{d}
\end{aligned}
$$

and hence (recalling $z_{m}$ from (2.1))

$$
\mathbb{P}_{m}^{(\theta)}\left(A_{1}\right)=z_{m}\left(e^{-\beta / \theta}\left(1+\frac{\beta}{\theta}\right)\right)^{d} .
$$



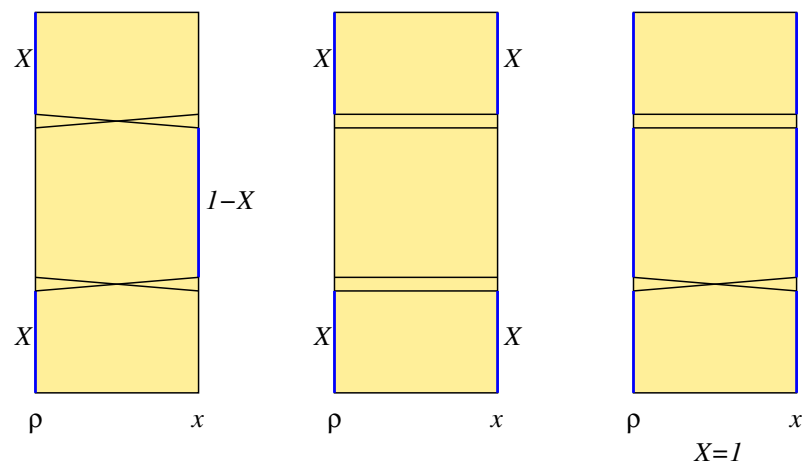

Fig. 3 Illustration of the possibilities for $\omega_{\rho x}$ on the event $A_{2}$. On $A_{2}^{\text {same }}$ there are two loops, one of which contains $(\rho, 0)$; on $A_{2}^{\operatorname{mix}}$ only one. The latter is thus more advantageous for long connections. The random variable $X$ has mean $\frac{2}{3}$

Similarly, since the $k$ children with links would need to be blocked from reaching distance $m-1$, we also have

$$
\begin{aligned}
\mathbb{P}_{m}^{(\theta)}\left(A_{1} \cap B_{m}^{\rho}\right) & =\frac{\theta}{Z_{m}} \sum_{k=0}^{d}\left(\begin{array}{l}
d \\
k
\end{array}\right)\left(e^{-\beta}\right)^{d-k}\left(e^{-\beta} \frac{\beta}{\theta}\right)^{k} Z_{m-1}^{d-k} \mathbb{E}_{m-1}\left[\theta^{\ell} \mathbb{I}_{B_{m-1}^{\rho}}\right]^{k} \\
& =z_{m}\left(e^{-\beta / \theta}\left(1+\zeta_{m-1} \frac{\beta}{\theta}\right)\right)^{d} .
\end{aligned}
$$

For the event $A_{2}$, we decompose it as $A_{2}=A_{2}^{\text {mix }} \cup A_{2}^{\text {same }}$, according as the 2 links from $\rho$ to $x$ are different sorts (crosses/double-bars) or the same (Fig 3). If we look at the restriction of $\omega$ to the link $\rho x$ only (i.e., at $\omega_{\rho x}$ ) then it has two loops on $A_{2}^{\text {same }}$ and a single loop on $A_{2}^{\text {mix }}$. Let us number the children of $x$ together with the children of $\rho$ excepting $x$ by $i=1, \ldots, 2 d-1$. Then we have that

$$
\ell=\sum_{i=1}^{2 d-1} \ell_{i}-k+ \begin{cases}1 & \text { on } A_{2}^{\text {mix }} \\ 2 & \text { on } A_{2}^{\text {same }}\end{cases}
$$

where $k$ denotes the total number of 1-links at $\rho$ and at $x$. To see this one may again imagine that the 1-links are placed last, one at a time. If $k=0$ then (2.8) holds due to our observation about $A_{2}^{\text {mix }}$ and $A_{2}^{\text {same }}$ above, if $k>0$ then each link we place merges two previously disjoint loops.

Let $\Lambda$ denote the loop in $\omega_{\rho x}$ containing $(\rho, 0)$, and let $\Lambda_{\rho}=\Lambda \cap(\{\rho\} \times[0,1])$ and $\Lambda_{x}=\Lambda \cap(\{x\} \times[0,1])$ denote the parts of $\Lambda$ at $\rho$ and at $x$, respectively. For $B_{m}^{\rho}$ to happen, children of $\rho$ which link to $\Lambda$ need to be blocked from distance $m-1$ and children of $x$ which link to $\Lambda$ need to be blocked from distance $m-2$; the remaining children of $\rho$ and $x$ do not need to be blocked. In particular, on $A_{2}^{\text {mix }}$ all children which link to either $\rho$ or $x$ need to be blocked. Write $A_{2}^{\operatorname{mix}}\left(x, k_{0}, k_{1}\right)$ for the event that (i) $\rho x$ supports one link of each sort, (ii) among the remaining children of $\rho$ exactly $k_{0}$ support 1 link and the rest 0 , and (iii) among the children of $x$ exactly $k_{1}$ support 1 link and the rest 0 . Using (2.8) with $k=k_{0}+k_{1}$ 
and a calculation similar to (2.7) we get

$$
\begin{aligned}
\mathbb{E}_{m}\left[\theta^{\ell} \mathbb{I}_{B_{m}^{\rho}} \mathbb{I}_{A_{2}^{\text {mix }}}\right]= & \sum_{x \sim \rho} \sum_{k_{0}=0}^{d-1} \sum_{k_{1}=0}^{d} \theta^{-k_{0}-k_{1}+1} \mathbb{E}_{m}\left[\theta^{\sum_{i} \ell_{i}} \mathbb{I}_{A_{2}^{\text {mix }}\left(x, k_{0}, k_{1}\right)} \mathbb{I}_{B_{m}^{\rho}}\right] \\
= & \theta Z_{m-1}^{d-1} Z_{m-2}^{d} \frac{d \beta^{2} e^{-\beta}}{2} 2 u(1-u)\left(e^{-\beta}\left(1+\frac{\beta}{\theta} \zeta_{m-1}\right)\right)^{d-1} \\
& \times\left(e^{-\beta}\left(1+\frac{\beta}{\theta} \zeta_{m-2}\right)\right)^{d} .
\end{aligned}
$$

For the case of $A_{2}^{\text {same }}$ we may start with a similar decomposition,

$$
\mathbb{E}_{m}\left[\theta^{\ell} \mathbb{I}_{B_{m}^{\rho}} \mathbb{I}_{A_{2}^{\text {same }}}\right]=\sum_{x \sim \rho} \sum_{k_{0}=0}^{d-1} \sum_{k_{1}=0}^{d} \theta^{-k_{0}-k_{1}+2} \mathbb{E}_{m}\left[\theta^{\sum_{i} \ell_{i}} \mathbb{I}_{A_{2}^{\text {same }}\left(x, k_{0}, k_{1}\right)} \mathbb{I}_{B_{m}^{\rho}}\right]
$$

where $A_{2}^{\text {same }}\left(x, k_{0}, k_{1}\right)$ is defined as $A_{2}^{\text {mix }}\left(x, k_{0}, k_{1}\right)$ except for requiring the two links supported by $\rho x$ to be of the same sort instead. Here we may then further consider the number $j_{0} \in\left\{0, \ldots, k_{0}\right\}$ of links with an endpoint in $\Lambda_{\rho}$ as well as the number $j_{1} \in\left\{0, \ldots, k_{1}\right\}$ of links with an endpoint in $\Lambda_{x}$. As mentioned above, these links need to be blocked, but the remaining do not. Recalling that the locations of links are uniform on $[0,1]$ this means that we obtain a factor $\left|\Lambda_{\rho}\right|$ (respectively $\left|\Lambda_{x}\right|$ ) for each of these $j_{0}$ (respectively, $j_{1}$ ) links, and hence

$$
\begin{aligned}
\mathbb{E}_{m}\left[\theta^{\ell} \mathbb{I}_{B_{m}^{\rho}} \mathbb{I}_{A_{2}^{\text {same }}}\right]= & \theta^{2} Z_{m-1}^{d-1} Z_{m-2}^{d} \frac{d \beta^{2} e^{-\beta}}{2}\left(u^{2}+(1-u)^{2}\right) \\
& \mathbb{E}\left[\left(e^{-\beta}\left(1+\frac{\beta}{\theta} \zeta_{m-1}\left|\Lambda_{\rho}\right|+\frac{\beta}{\theta}\left(1-\left|\Lambda_{\rho}\right|\right)\right)\right)^{d-1}\right. \\
& \left.\times\left(e^{-\beta}\left(1+\frac{\beta}{\theta} \zeta_{m-2}\left|\Lambda_{x}\right|+\frac{\beta}{\theta}\left(1-\left|\Lambda_{x}\right|\right)\right)\right)^{d}\right] .
\end{aligned}
$$

Here we have simply written $\mathbb{E}[\cdot]$ for $\mathbb{E}_{m}\left[\cdot \mid A_{2}\right]$, this expectation is over the choice of crosses or double-bars and over the lengths $\left|\Lambda_{\rho}\right|$ and $\left|\Lambda_{x}\right|$ only.

We note here that the joint expectations of $\left|\Lambda_{\rho}\right|$ and $\left|\Lambda_{x}\right|$ may be computed explicitly. Indeed, as illustrated in Fig. 3, there is a random variable $X$ such that $\Lambda_{\rho}$ and $\Lambda_{x}$ have respective lengths $X$ and $1-X$ in the case of two crosses; $X$ and $X$ in the case of two double-bars; and $\left|\Lambda_{\rho}\right|=\left|\Lambda_{X}\right|=X=1$ in the case of a mixture. We have the following:

Lemma $2.2 \mathbb{E}_{m}\left[X \mid A_{2}^{\text {same }}\right]=\frac{2}{3}$.

Proof The conditional distribution of $X$ given $A_{2}^{\text {same }}$ is that of that of $1-\left(U_{1} \vee U_{2}-U_{1} \wedge U_{2}\right)$ where $U_{1}, U_{2}$ are independent random variables, uniformly distributed on $[0,1]$. Using that $U_{1} \wedge U_{2}=U_{1}+U_{2}-U_{1} \vee U_{2}$ and $\mathbb{P}\left(U_{1} \vee U_{2} \leq t\right)=t^{2}$ for $t \in[0,1]$ gives the result.

At this point, let us mention the following asymptotics, which will be useful several times: if $\sigma=O\left(d^{-1}\right)$ and $x \in \mathbb{R}$ then we have

$$
\begin{aligned}
& \left(e^{-\beta / \theta}\left(1+\frac{\beta}{\theta}-\sigma x \frac{\beta}{\theta}\right)\right)^{d}=1-\frac{1}{d}(1 / 2+x \sigma d) \\
& \quad+\frac{1}{d^{2}}\left(1 / 3-\alpha+x \sigma d-\alpha x \sigma d+\frac{1}{2}(1 / 2+x \sigma d)^{2}\right)+O\left(d^{-3}\right) .
\end{aligned}
$$


To compute $\mathbb{P}\left(A_{2}\right)$ we may remove the enforcement of $B_{m}^{\rho}$ in (2.9) and (2.10) by setting $\zeta_{m-1}$ and $\zeta_{m-2}$ to 1 and summing the results together, giving

$$
\begin{aligned}
\mathbb{P}_{m}^{(\theta)}\left(A_{2}\right) & =z_{m} z_{m-1} \frac{d \beta^{2} e^{-\beta / \theta}}{2 \theta}\left(e^{-\beta / \theta}\left(1+\frac{\beta}{\theta}\right)\right)^{2 d-1}\left(2 u(1-u)+\theta\left(u^{2}+(1-u)^{2}\right)\right) \\
& =z_{m} z_{m-1} \frac{\theta}{2 d}\left[1-\frac{1}{d}\right]\left(2 u(1-u)+\theta\left(u^{2}+(1-u)^{2}\right)+O\left(d^{-2}\right)\right) .
\end{aligned}
$$

For the last step we used (2.11) to first order, and that

$$
\frac{d \beta^{2} e^{-\beta / \theta}}{2 \theta}=\frac{\theta}{2 d}+O\left(d^{-2}\right)
$$

\subsection{Stochastic Domination}

In some estimates we will want to approximate the complicated measure $\mathbb{P}_{m}^{(\theta)}(\cdot)$, which involves counting loops, by some simpler measure. For this we use stochastic domination. Let us define $\beta^{+}=(\beta \theta) \vee(\beta / \theta)$. Also let us define $\mathbb{E}_{m}^{+}$in the same way as $\mathbb{E}_{m}$ but with $\beta$ replaced by $\beta^{+}$; thus the links form independent Poisson processes with rate $\beta^{+}$. We say that an event $A$ is increasing if it cannot be destroyed by adding more links; examples of increasing events include $A_{1}^{c}$ and $\left(A_{1} \cup A_{2}\right)^{c}$ where $A_{1}$ and $A_{2}$ are as defined above. Stochastic domination tells us that

$$
A \text { increasing } \Rightarrow \mathbb{P}_{m}^{(\theta)}(A) \leq \mathbb{P}_{m}^{+}(A) \text {. }
$$

Proof of (2.14) We apply [8, Thm. 1.1]. Note that $\mathbb{P}_{m}^{(\theta)} \ll \mathbb{P}_{m}^{+}$and the density $f(\omega)=\frac{d \mathbb{P}_{m}^{(\theta)}}{d \mathbb{P}_{m}^{+}} \propto$ $\theta^{\ell(\omega)}\left(\frac{\beta}{\beta^{+}}\right)^{|\omega|}$ where $|\omega|$ denotes the number of links. Let $\omega^{\prime}$ be obtained from $\omega$ by adding a single link. This link either splits a loop, merges two loops, or does not change the number of loops, hence

$$
\frac{f\left(\omega^{\prime}\right)}{f(\omega)}=\theta^{\ell\left(\omega^{\prime}\right)-\ell(\omega)} \frac{\beta}{\beta^{+}} \in\left\{\frac{\beta \theta}{\beta^{+}}, \frac{\beta / \theta}{\beta^{+}}, \frac{\beta}{\beta^{+}}\right\} .
$$

The result follows since all three possible values are $\leq 1$.

An immediate consequence of (2.14) is that there is some constant $c>0$ such that

$$
\mathbb{P}_{m}^{(\theta)}\left(A_{1}^{c} \cap A_{2}^{c}\right) \leq c / d^{2} \text { for all } m, d \geq 1 .
$$

We now deduce some information about the asymptotic behaviour of the numbers $z_{m}=$ $e^{-d \beta(1-1 / \theta)} \theta Z_{m-1}^{d} / Z_{m}$. We write

$$
\begin{aligned}
& q=q(\theta, u)=\frac{\theta}{2}\left(2 u(1-u)+\theta\left(u^{2}+(1-u)^{2}\right)\right) \\
& r=r(\theta, u)=2 \theta u(1-u)+\frac{1}{2} \theta^{2}\left(u^{2}+\frac{4}{3}(1-u)^{2}\right)
\end{aligned}
$$

so that $\alpha_{*}=1+q-r$.

Proposition 2.3 There is a constant $C$ and there are functions $\varepsilon_{m}^{(j)}(d), j \in\{1,2,3\}$, satisfying

$$
\left|\varepsilon_{m}^{(j)}(d)\right| \leq C / d^{2} \text { for all } m, d \geq 1,
$$

such that

$$
z_{m}=1-\frac{1}{d}(q-1 / 2)+\varepsilon_{m}^{(1)}(d)
$$

and

$$
z_{m}\left(1+\frac{1}{d}(q-1 / 2)+\varepsilon_{m}^{(2)}(d)\right)=1-\varepsilon_{m}^{(3)}(d) .
$$


Proof Note that (2.19) and (2.20) are equivalent, hence one may proceed by induction on $m$, proving (2.20) with the induction hypothesis provided by (2.19). For the base case $m=1$ one may establish (2.19) directly, splitting into the cases $A_{1}, A_{2}$ and $\left(A_{1} \cup A_{2}\right)^{c}$ to get

$$
Z_{1}=\theta^{d+1} e^{-d \beta(1-1 / \theta)}\left[\left(e^{-\beta / \theta}\left(1+\frac{\beta}{\theta}\right)\right)^{d}+q(\theta, u) \frac{d \beta^{2} e^{-\beta / \theta}}{\theta^{2}}\left(e^{-\beta / \theta}\left(1+\frac{\beta}{\theta}\right)\right)^{d-1}+\varepsilon_{1}(d)\right],
$$

where $0 \leq \varepsilon_{1}(d) \leq e^{d \beta(1-1 / \theta)} \mathbb{P}_{1}\left(A_{1}^{c} \cap A_{2}^{c}\right)$ satisfies (2.18).

For $m>1$, write $\varepsilon_{m}^{(3)}(d)=\mathbb{P}_{m}^{(\theta)}\left(A_{1}^{c} \cap A_{2}^{c}\right)$, this satisfies (2.18) by (2.15). From the expressions (2.6) and (2.12) we have

$$
\begin{aligned}
1 & =\mathbb{P}_{m}^{(\theta)}\left(A_{1}\right)+\mathbb{P}_{m}^{(\theta)}\left(A_{2}\right)+\mathbb{P}_{m}^{(\theta)}\left(A_{1}^{c} \cap A_{2}^{c}\right) \\
& =z_{m}\left(e^{-\beta / \theta}\left(1+\frac{\beta}{\theta}\right)\right)^{d}+z_{m} z_{m-1} \frac{d \beta^{2} e^{-\beta / \theta}}{\theta^{2}}\left(e^{-\beta / \theta}\left(1+\frac{\beta}{\theta}\right)\right)^{2 d-1} q(\theta, u)+\varepsilon_{m}^{(3)}(d)
\end{aligned}
$$

Hence, using the asymptotics (2.11) and (2.13),

$$
1-\varepsilon_{m}^{(3)}(d)=z_{m}\left[1-\frac{1}{2 d}+z_{m-1} \frac{1}{d}\left(1-\frac{1}{d}\right) q+\varepsilon^{(4)}(d)\right]
$$

for a function $\varepsilon^{(4)}(d)$ not depending on $m$ but otherwise satisfying the bounds (2.18). Using the induction hypothesis we get

$$
1-\varepsilon_{m}^{(3)}(d)=z_{m}\left[1+\frac{1}{d}\left(q-\frac{1}{2}\right)+\varepsilon_{m}^{(2)}(d)\right],
$$

where

$$
\varepsilon_{m}^{(2)}(d)=\varepsilon^{(4)}(d)-\frac{q}{d^{2}}\left(q+\frac{1}{2}\right)+\frac{q}{d^{3}}\left(q-\frac{1}{2}\right)+\frac{q}{d}\left(1-\frac{1}{d}\right) \varepsilon_{m-1}^{(1)}(d)
$$

is easily seen to satisfy (2.18).

Remark 2.4 From the proposition it follows that

$$
z_{m} z_{m-1}=1+O\left(d^{-1}\right)
$$

where the $O(\cdot)$ is uniform in $m$.

We now turn to the details of the proof of Proposition 2.1.

\subsection{Proof of the Lower Bound (2.2)}

We have from the definition $\sigma_{m}=1-\mathbb{P}_{m}^{(\theta)}\left(B_{m}^{\rho}\right)$ that

$$
\sigma_{m} \geq \mathbb{P}_{m}^{(\theta)}\left(A_{1}\right)-\mathbb{P}_{m}^{(\theta)}\left(B_{m}^{\rho} \cap A_{1}\right)+\mathbb{P}_{m}^{(\theta)}\left(A_{2}\right)-\mathbb{P}_{m}^{(\theta)}\left(B_{m}^{\rho} \cap A_{2}\right),
$$

where we have simply bounded the remaining difference involving the event $\left(A_{1} \cup A_{2}\right)^{c}$ from below by 0 . Consider first the terms involving $A_{1}$. From (2.6) and (2.7), bounding $\sigma_{m-1} \geq \tilde{\sigma}_{m-1}$, and using the asymptotics (2.11) as well as the estimates Proposition 2.3 on $z_{m}$ we get

$$
\begin{aligned}
\mathbb{P}_{m}^{(\theta)} & \left(A_{1}\right)-\mathbb{P}_{m}^{(\theta)}\left(B_{m}^{\rho} \cap A_{1}\right) \geq z_{m}\left(\tilde{\sigma}_{m-1}-\frac{\tilde{\sigma}_{m-1}}{d}(3 / 2-\alpha)-\frac{1}{2} \tilde{\sigma}_{m-1}^{2}+O\left(d^{-3}\right)\right) \\
& =\left(1-\frac{1}{d}(q-1 / 2)\right)\left(\tilde{\sigma}_{m-1}-\frac{\tilde{\sigma}_{m-1}}{d}(3 / 2-\alpha)-\frac{1}{2} \tilde{\sigma}_{m-1}^{2}\right)+O\left(d^{-3}\right) \\
& =\tilde{\sigma}_{m-1}+\frac{\tilde{\sigma}_{m-1}}{d}(\alpha-q-1)-\frac{1}{2} \tilde{\sigma}_{m-1}^{2}+O\left(d^{-3}\right) .
\end{aligned}
$$


Now consider the terms involving $A_{2}$. Using that $\zeta_{m-1}, \zeta_{m-2} \leq 1-\tilde{\sigma}_{m-1}$, as well as the asymptotics (2.11) to order $d^{-1}$, we deduce from (2.9) that

$$
\begin{aligned}
\mathbb{P}_{m}^{(\theta)}\left(B_{m}^{\rho} \cap A_{2}^{\mathrm{mix}}\right) & \leq z_{m} z_{m-1} \frac{d \beta^{2} e^{-\beta / \theta}}{2 \theta} 2 u(1-u)\left(e^{-\beta / \theta}\left(1+\frac{\beta}{\theta}-\tilde{\sigma}_{m-1} \frac{\beta}{\theta}\right)\right)^{2 d-1} \\
& =z_{m} z_{m-1} \frac{\theta}{2 d}\left[\left(1-\frac{1}{d}\right) 2 u(1-u)-\tilde{\sigma}_{m-1} 4 u(1-u)+O\left(d^{-2}\right)\right]
\end{aligned}
$$

and from (2.10) that

$$
\begin{aligned}
\mathbb{P}_{m}^{(\theta)}\left(B_{m}^{\rho} \cap A_{2}^{\text {same }}\right) \leq & z_{m} z_{m-1} \frac{d \beta^{2} e^{-\beta / \theta}}{2}\left(u^{2}+(1-u)^{2}\right) \\
& \mathbb{E}\left[\left(e^{-\beta / \theta}\left(1+\frac{\beta}{\theta}-\tilde{\sigma}_{m-1}\left|\Lambda_{\rho}\right| \frac{\beta}{\theta}\right)\right)^{d-1}\left(e^{-\beta / \theta}\left(1+\frac{\beta}{\theta}-\tilde{\sigma}_{m-1}\left|\Lambda_{x}\right| \frac{\beta}{\theta}\right)\right)^{d}\right] \\
= & z_{m} z_{m-1} \frac{\theta^{2}}{2 d}\left(u^{2}+(1-u)^{2}\right)\left[\left(1-\frac{1}{d}\right)-\tilde{\sigma}_{m-1} \mathbb{E}\left(\left|\Lambda_{\rho}\right|+\left|\Lambda_{x}\right|\right)+O\left(d^{-2}\right)\right] \\
= & z_{m} z_{m-1} \frac{\theta^{2}}{2 d}\left[\left(1-\frac{1}{d}\right)\left(u^{2}+(1-u)^{2}\right)-\tilde{\sigma}_{m-1}\left(u^{2}+\frac{4}{3}(1-u)^{2}\right)+O\left(d^{-2}\right)\right] .
\end{aligned}
$$

Here we used the properties of $\left|\Lambda_{\rho}\right|$ and $\left|\Lambda_{x}\right|$ stated below (2.10) (see also Fig. 3). Using also (2.12) and (2.21) we get

$$
\begin{aligned}
& \mathbb{P}_{m}^{(\theta)}\left(A_{2}\right)-\mathbb{P}_{m}^{(\theta)}\left(B_{m}^{\rho} \cap A_{2}\right) \\
& \geq z_{m} z_{m-1} \frac{\theta}{2 d}\left\{2 u(1-u)\left(\left[1-\frac{1}{d}\right]-\left[1-\frac{1}{d}-2 \tilde{\sigma}_{m-1}\right]\right)\right. \\
& \left.\quad+\theta\left(u^{2}+(1-u)^{2}\right)\left(\left[1-\frac{1}{d}\right]-\left[1-\frac{1}{d}-\tilde{\sigma}_{m-1} \frac{u^{2}+\frac{4}{3}(1-u)^{2}}{u^{2}+(1-u)^{2}}\right]\right)+O\left(d^{-2}\right)\right\} \\
& =r(\theta, u) \frac{\tilde{\sigma}_{m-1}}{d}+O\left(d^{-3}\right),
\end{aligned}
$$

where $r$ is defined in (2.17). Putting this together in (2.22) gives

$$
\sigma_{m} \geq \tilde{\sigma}_{m-1}+\frac{\tilde{\sigma}_{m-1}}{d}(\alpha-[1+q-r])-\frac{1}{2} \tilde{\sigma}_{m-1}^{2}+O\left(d^{-3}\right) .
$$

Since $\alpha_{*}=1+q-r$ this gives (2.2).

\subsection{Proof of the Upper Bound (2.3)}

Write $\Sigma_{m}^{\rho}$ for the complement of $B_{m}^{\rho}$, so that $\sigma_{m}=\mathbb{P}_{m}^{(\theta)}\left(\Sigma_{m}^{\rho}\right)$. Clearly

$$
\sigma_{m}=\mathbb{P}_{m}^{(\theta)}\left(A_{1} \cap \Sigma_{m}^{\rho}\right)+\mathbb{P}_{m}^{(\theta)}\left(A_{2} \cap \Sigma_{m}^{\rho}\right)+\mathbb{P}_{m}^{(\theta)}\left(A_{1}^{c} \cap A_{2}^{c} \cap \Sigma_{m}^{\rho}\right) .
$$

The following will be proved at the end of this section:

Proposition 2.5 For all d large enough there is a constant $C$ such that

$$
\mathbb{P}_{m}^{(\theta)}\left(A_{1}^{c} \cap A_{2}^{c} \cap \Sigma_{m}^{\rho}\right) \leq \frac{C}{d^{2}}\left(\sigma_{m-1} \vee \sigma_{m-2}\right)
$$


Before proving this we show how to deduce (2.3). We have by taking the difference of the expressions (2.6) and (2.7) that

$$
\begin{aligned}
\mathbb{P}_{m}^{(\theta)}\left(A_{1} \cap \Sigma_{m}^{\rho}\right) & =z_{m}\left\{\left(e^{-\beta / \theta}\left(1+\frac{\beta}{\theta}\right)\right)^{d}-\left(e^{-\beta / \theta}\left(1+\frac{\beta}{\theta}-\frac{\beta}{\theta} \sigma_{m-1}\right)\right)^{d}\right\} \\
& =z_{m}\left(e^{-\beta / \theta}\left(1+\frac{\beta}{\theta}\right)\right)^{d}\left\{1-\left(1-\frac{\frac{\beta}{\theta} \sigma_{m-1}}{1+\beta / \theta}\right)^{d}\right\} \\
& \leq z_{m}\left(e^{-\beta / \theta}\left(1+\frac{\beta}{\theta}\right)\right)^{d} \frac{d \beta}{\theta}\left(1+\frac{\beta}{\theta}\right)^{-1} \sigma_{m-1} .
\end{aligned}
$$

In the last step we used the concavity of the function $f(x)=1-(1-x)^{d}$ to bound $f(x) \leq x f^{\prime}(0)$.

Similarly using (2.9) and concavity of $f(x, y)=1-(1-x)^{d-1}(1-y)^{d}$ (for $d \geq 3$ ),

$$
\begin{aligned}
\mathbb{P}_{m}^{(\theta)}\left(\Sigma_{m}^{\rho} \cap A_{2}^{\text {mix }}\right)= & z_{m} z_{m-1} \frac{d \beta^{2} e^{-\beta / \theta}}{2} \frac{2}{\theta} u(1-u)\left(e^{-\beta / \theta}\left(1+\frac{\beta}{\theta}\right)\right)^{2 d-1} \\
& \times\left\{1-\left(1-\frac{\frac{\beta}{\theta} \sigma_{m-1}}{1+\frac{\beta}{\theta}}\right)^{d-1}\left(1-\frac{\frac{\beta}{\theta} \sigma_{m-2}}{1+\frac{\beta}{\theta}}\right)^{d}\right\} \\
\leq & z_{m} z_{m-1} \frac{d \beta^{2} e^{-\beta / \theta}}{2} \frac{2}{\theta} u(1-u)\left(e^{-\beta / \theta}\left(1+\frac{\beta}{\theta}\right)\right)^{2 d-1} \\
& \times \frac{\beta}{\theta}\left(1+\frac{\beta}{\theta}\right)^{-1}\left\{(d-1) \sigma_{m-1}+d \sigma_{m-2}\right\} \\
\leq & z_{m} z_{m-1} \frac{d \beta^{2} e^{-\beta / \theta}}{2}\left(e^{-\beta / \theta}\left(1+\frac{\beta}{\theta}\right)\right)^{2 d-1} \\
& \times \frac{d \beta}{\theta}\left(1+\frac{\beta}{\theta}\right)^{-1} \frac{2}{\theta} u(1-u)\left(\sigma_{m-1}+\sigma_{m-2}\right) .
\end{aligned}
$$

The same argument applied to (2.10) gives (with the notation $\mathbb{E}$ used there and using Lemma 2.2)

$$
\begin{aligned}
\mathbb{P}_{m}^{(\theta)}\left(\Sigma_{m}^{\rho} \cap A_{2}^{\text {same }}\right)= & z_{m} z_{m-1} \frac{d \beta^{2} e^{-\beta / \theta}}{2}\left(u^{2}+(1-u)^{2}\right)\left(e^{-\beta / \theta}\left(1+\frac{\beta}{\theta}\right)\right)^{2 d-1} \\
& \cdot \mathbb{E}\left[1-\left(1-\frac{\frac{\beta}{\theta} \sigma_{m-1}\left|\Lambda_{\rho}\right|}{1+\frac{\beta}{\theta}}\right)^{d-1}\left(1-\frac{\frac{\beta}{\theta} \sigma_{m-2}\left|\Lambda_{x}\right|}{1+\frac{\beta}{\theta}}\right)^{d}\right] \\
\leq & z_{m} z_{m-1} \frac{d \beta^{2} e^{-\beta / \theta}}{2}\left(u^{2}+(1-u)^{2}\right)\left(e^{-\beta / \theta}\left(1+\frac{\beta}{\theta}\right)\right)^{2 d-1} \frac{\beta}{\theta}\left(1+\frac{\beta}{\theta}\right)^{-1} \\
& \cdot\left\{(d-1) \sigma_{m-1} \mathbb{E}\left|\Lambda_{\rho}\right|+d \sigma_{m-2} \mathbb{E}\left|\Lambda_{x}\right|\right\} \\
\leq & z_{m} z_{m-1} \frac{d \beta^{2} e^{-\beta / \theta}}{2}\left(e^{-\beta / \theta}\left(1+\frac{\beta}{\theta}\right)\right)^{2 d-1} \frac{d \beta}{\theta}\left(1+\frac{\beta}{\theta}\right)^{-1} \\
& \cdot\left\{\sigma_{m-1} \frac{2}{3}\left(u^{2}+(1-u)^{2}\right)+\sigma_{m-2}\left(\frac{1}{3} u^{2}+\frac{2}{3}(1-u)^{2}\right)\right\}
\end{aligned}
$$

Using Proposition 2.3 to estimate $z_{m}$, the asymptotics (2.11), as well as $\frac{d \beta}{\theta}\left(1+\frac{\beta}{\theta}\right)^{-1}=$ $1+\frac{\alpha-1}{d}+O\left(d^{-2}\right)$ we see that the right-hand side of (2.26) satisfies

$$
z_{m}\left(e^{-\beta / \theta}\left(1+\frac{\beta}{\theta}\right)\right)^{d} \frac{d \beta}{\theta}\left(1+\frac{\beta}{\theta}\right)^{-1} \sigma_{m-1}=\left(1+\frac{\alpha-(1+q)}{d}+O\left(d^{-2}\right)\right) \sigma_{m-1},
$$

where $q=q(\theta, u)$ was defined in (2.16). Similarly, using (2.13) and (2.21), in the righthand-sides of (2.27) and (2.28) we have the factors

$$
z_{m} z_{m-1} \frac{d \beta^{2} e^{-\beta / \theta}}{2}\left(e^{-\beta / \theta}\left(1+\frac{\beta}{\theta}\right)\right)^{2 d-1} \frac{d \beta}{\theta}\left(1+\frac{\beta}{\theta}\right)^{-1}=\frac{\theta^{2}}{2 d}\left(1+O\left(d^{-1}\right)\right) .
$$


Hence, bounding also $\sigma_{m-1}$ and $\sigma_{m-2}$ by their maximum, we have that

$$
\begin{aligned}
\sigma_{m} \leq & \left(\sigma_{m-1} \vee \sigma_{m-2}\right)\left[1+\frac{\alpha-(1+q)}{d}+\frac{\theta^{2}}{2 d}\left(\frac{4}{\theta} u(1-u)+\frac{2}{3}\left(u^{2}+(1-u)^{2}\right)+\frac{1}{3} u^{2}\right.\right. \\
& \left.\left.+\frac{2}{3}(1-u)^{2}\right)+O\left(d^{-2}\right)\right]+\mathbb{P}_{m}^{(\theta)}\left(A_{1}^{c} \cap A_{2}^{c} \cap \Sigma_{m}^{\rho}\right) \\
= & \left(\sigma_{m-1} \vee \sigma_{m-2}\right)\left[1+\frac{\alpha-(1+q-r)}{d}+O\left(d^{-2}\right)\right]+\mathbb{P}_{m}^{(\theta)}\left(A_{1}^{c} \cap A_{2}^{c} \cap \Sigma_{m}^{\rho}\right),
\end{aligned}
$$

where $r=r(\theta, u)$ was defined in (2.17). In the above, all $O\left(d^{-2}\right)$ terms are uniform in $m$. Since $1+q-r=\alpha_{*}$ we see that (2.3) follows once we prove Proposition 2.5.

In the following argument we will examine the subtree $\breve{T}$ of $T_{m}$ which contains the root and is spanned by edges supporting at least two links. In $\check{T}$, the loop-structure is very complicated and we will not attempt to keep track of it. Instead we use that $\breve{T}$ is likely to be small, and that a loop exiting it must do so across an edge supporting exactly one link, which is a simpler situation to analyze. Roughly speaking, the enforcement of the event $A_{1}^{c} \cap A_{2}^{c}$ will give rise to the factor $d^{-2}$, and the requirement that the loop exits $\breve{T}$ will give a factor $\sigma_{m-k}$ for some $k \geq 1$, which can then be bounded in terms of $\sigma_{m-1} \vee \sigma_{m-2}$. The details are quite technical.

Proof of Proposition 2.5 We begin by defining $\check{T}$ carefully: we let $\breve{T}$ be the (random) subtree of $T_{m}$ containing

(1) The root $\rho$

(2) Any vertex in generation 1 with $\geq 2$ links to $\rho$,

(3) In general, any vertex in generation $k$ with $\geq 2$ links to some vertex of $\check{T}$ in generation $k-1$.

Note that $A_{1}^{c} \cap A_{2}^{c}$ is precisely the event that $\check{T}$ has at least two edges. Let $V_{k}(\check{T})$ denote the set of vertices in $\breve{T}$ in generation $k$. For $x$ a vertex of $\check{T}, x \notin V_{m}(\breve{T})$, let $d_{x}$ denote its number of descendants not in $\breve{T}$. Thus $x$ has $d_{x}$ outgoing edges carrying only 0 or 1 links of $\omega$. For $0 \leq k \leq m-1$ we let $\mathcal{E}_{k}$ denote the set of outgoing edges from generation $k$ (to generation $k+1)$ which carry precisely 1 link.

Note that if the loop of $(\rho, 0)$ reaches generation $m$ then either it reaches generation $m$ within $\check{T}$, or it passes some link of $\cup_{k=0}^{m-1} \mathcal{E}_{k}$. Let us by convention set $\sigma_{-1}=1$ and $\left|\mathcal{E}_{m}\right|=\left|V_{m}(\check{T})\right|$. We claim that

$$
\mathbb{P}_{m}^{(\theta)}\left(A_{1}^{c} \cap A_{2}^{c} \cap \Sigma_{m}^{\rho}\right) \leq \sum_{k=0}^{m} \sigma_{m-k-1} \mathbb{E}_{m}^{(\theta)}\left[\left|\mathcal{E}_{k}\right| \mathbb{I}_{A_{1}^{c} \cap A_{2}^{c}}\right] .
$$

Intuitively, this is because if the loop exits $\breve{T}$ through some edge in $\mathcal{E}_{k}$, then it has distance $m-k-1$ left to go to reach the $m$ th generation of $T_{m}$. A detailed justification of (2.29) requires dealing with the dependencies caused by the factor $\theta^{\ell}$.

To do this, let us introduce the following notation. First, let $\check{\omega}$ denote the restriction of $\omega$ to $\check{T}$. Next, let $\partial^{+} \check{T}$ denote the set of vertices $y \in T_{m} \backslash \check{T}$ whose parent belongs to $\check{T}$, and write $\omega_{y}$ for the restriction of $\omega$ to the subtree rooted at $y$. For simplicity, in the rest of this proof we simply write $\mathbb{E}$ for $\mathbb{E}_{m}$. We will make use of the fact that, given $\check{\omega}$, the random collections $\left(\mathcal{E}_{j}\right)_{j=0}^{m-1}$ and $\left(\omega_{y}\right)_{y \in \partial^{+} \check{T}}$ are conditionally independent under $\mathbb{E}$. This implies that for three functions

$$
F_{1}(\check{\omega}), \quad F_{2}\left(\check{\omega},\left(\mathcal{E}_{j}\right)_{j=0}^{m-1}\right), \quad F_{3}\left(\check{\omega},\left(\omega_{y}\right)_{y \in \partial^{+}} \check{T}\right)
$$


we have

$$
\begin{aligned}
\mathbb{E} & {\left[F_{1}(\check{\omega}) F_{2}\left(\check{\omega},\left(\mathcal{E}_{j}\right)_{j=0}^{m-1}\right) F_{3}\left(\check{\omega},\left(\omega_{y}\right)_{y \in \partial^{+} \check{T}}\right)\right] } \\
& =\mathbb{E}\left[F_{1}(\check{\omega}) \mathbb{E}\left[F_{2}\left(\check{\omega},\left(\mathcal{E}_{j}\right)_{j=0}^{m-1}\right) \mid \check{\omega}\right] \mathbb{E}\left[F_{3}\left(\check{\omega},\left(\omega_{y}\right)_{y \in \partial^{+}} \check{T}\right) \mid \check{\omega}\right]\right] .
\end{aligned}
$$

Note that we have the decomposition (similar to (2.5))

$$
\ell=\check{\ell}+\sum_{j=0}^{m-1}\left[\sum_{x \in V_{j}(\check{T})} \sum_{i=1}^{d_{x}} \ell_{i}^{(x)}-\left|\mathcal{E}_{j}\right|\right],
$$

where $\check{\ell}$ denotes the number of loops in the configuration $\check{\omega}$, and $\ell_{i}^{(x)}$ denotes the number of loops in the subtree rooted at the $i$ th descendant of $x$ not belonging to $\breve{T}$ (in some numbering of these descendants). Hence

$$
\theta^{\ell}=\theta^{\check{\ell}}\left(\prod_{j=0}^{m-1} \theta^{-\left|\mathcal{E}_{j}\right|}\right)\left(\prod_{j=0}^{m-1} \prod_{x \in V_{j}(\check{T})} \prod_{i=1}^{d_{x}} \theta^{\ell_{i}^{(x)}}\right)
$$

is a factorization into three functions as in (2.30). Turning to (2.29), by considering the possibilities that either $\breve{T}$ reaches generation $m$ (meaning $V_{m}(\check{T}) \neq \varnothing$ ) or that loop of $(\rho, 0)$ passes some edge $e \in \cup_{k=0}^{m-1} \mathcal{E}_{k}$, we have

$$
\begin{aligned}
& \mathbb{P}_{m}^{(\theta)}\left(A_{1}^{c} \cap A_{2}^{c} \cap \Sigma_{m}^{\rho}\right) \leq \mathbb{E}_{m}^{(\theta)}\left[\left|V_{m}(\check{T})\right| \mathbb{I}_{A_{1}^{c} \cap A_{2}^{c}}\right] \\
& +\sum_{e} \sum_{k=0}^{m-1} \mathbb{P}_{m}^{(\theta)}\left(A_{1}^{c} \cap A_{2}^{c} \cap\left\{e \in \mathcal{E}_{k}\right\} \cap\left\{\left(e^{+}, t^{+}\right) \leftrightarrow m\right\}\right],
\end{aligned}
$$

where the first sum is over all edges $e$ of $T_{m}$, and $\left\{\left(e^{+}, t^{+}\right) \leftrightarrow m\right\}$ denotes the event that the further (from $\rho$ ) endpoint $\left(e^{+}, t^{+}\right)$of the unique link at $e$ lies in a loop of $\omega_{e^{+}}$reaching the $m$ th generation of $T_{m}$. Applying (2.31) and (2.32) we have

$$
\begin{aligned}
& \mathbb{P}_{m}^{(\theta)}\left(A_{1}^{c} \cap A_{2}^{c} \cap\left\{e \in \mathcal{E}_{k}\right\} \cap\left\{\left(e^{+}, t^{+}\right) \leftrightarrow m\right\}\right] \\
& \quad=\frac{1}{Z_{m}} \mathbb{E}\left[\mathbb{I}_{A_{1}^{c} \cap A_{2}^{c}} \dot{\ell}^{\check{\ell}} \mathbb{E}\left[\mathbb{I}\left\{e \in \mathcal{E}_{k}\right\} \prod_{j=0}^{m-1} \theta^{-\left|\mathcal{E}_{j}\right|} \mid \check{T}\right] \sigma_{m-k-1} \prod_{j=0}^{m-1} \prod_{x \in V_{j}(\check{T})} Z_{m-j-1}^{d_{x}}\right] .
\end{aligned}
$$

Taking out the factor $\sigma_{m-k-1}$, applying (2.31) again in reverse, and putting back into (2.33), we obtain (2.29).

We proceed by bounding the expectations

$$
\mathbb{E}_{m}^{(\theta)}\left[\left|\mathcal{E}_{k}\right| \mathbb{I}_{A_{1}^{c} \cap A_{2}^{c}}\right]=\frac{1}{Z_{m}} \mathbb{E}\left[\theta^{\ell}\left|\mathcal{E}_{k}\right| \mathbb{I}_{A_{1}^{c} \cap A_{2}^{c}}\right] .
$$

Arguing as above we get:

$$
\mathbb{E}\left[\theta^{\ell}\left|\mathcal{E}_{k}\right| \mathbb{I}_{A_{1}^{c} \cap A_{2}^{c}}\right]=\mathbb{E}\left[\mathbb{I}_{A_{1}^{c} \cap A_{2}^{c}} \theta^{\check{\ell}} \prod_{j=0}^{m-1} \prod_{x \in V_{j}(\check{T})} Z_{m-j-1}^{d_{x}} \mathbb{E}\left[\left|\mathcal{E}_{k}\right| \prod_{j=0}^{m-1} \theta^{-\left|\mathcal{E}_{j}\right|} \mid \check{T}\right]\right] .
$$


The $\left|\mathcal{E}_{j}\right|$ are conditionally independent given $\check{T}$, hence

$$
\mathbb{E}\left[\left|\mathcal{E}_{k}\right| \prod_{j=0}^{m-1} \theta^{-\left|\mathcal{E}_{j}\right|} \mid \check{T}\right]=\mathbb{E}\left[\left|\mathcal{E}_{k}\right| \theta^{-\left|\mathcal{E}_{k}\right|} \mid \check{T}\right] \prod_{j \neq k} \mathbb{E}\left[\theta^{-\left|\mathcal{E}_{j}\right|} \mid \check{T}\right] .
$$

Let $p_{i}=e^{-\beta} \beta^{i} / i$ ! denote the probabilities of a Poisson $(\beta)$ random variable. Direct computation gives

$$
\mathbb{E}\left[\theta^{-\left|\mathcal{E}_{k}\right|} \mid \check{T}\right]=\prod_{x \in V_{k}(\check{T})}\left(\frac{p_{0}+p_{1} / \theta}{p_{0}+p_{1}}\right)^{d_{x}}
$$

and (e.g. by differentiating the previous expression)

$$
\mathbb{E}\left[\left|\mathcal{E}_{k}\right| \theta^{-\left|\mathcal{E}_{k}\right|} \mid \check{T}\right]=\frac{p_{1} / \theta}{p_{0}+p_{1} / \theta}\left(\sum_{x \in V_{k}(\check{T})} d_{x}\right) \prod_{x \in V_{k}(\check{T})}\left(\frac{p_{0}+p_{1} / \theta}{p_{0}+p_{1}}\right)^{d-d_{x}} .
$$

Hence

$$
\frac{\mathbb{E}\left[\left|\mathcal{E}_{k}\right| \theta^{-\left|\mathcal{E}_{k}\right|} \mid \check{T}\right]}{\mathbb{E}\left[\theta^{-\left|\mathcal{E}_{k}\right|} \mid \check{T}\right]} \leq \frac{d p_{1} / \theta}{p_{0}+p_{1} / \theta}\left|V_{k}(\check{T})\right|
$$

and

$$
\begin{aligned}
& \mathbb{E}\left[\theta^{\ell}\left|\mathcal{E}_{k}\right| \mathbb{I}_{A_{1}^{c} \cap A_{2}^{c}}\right] \\
& \leq \frac{d p_{1} / \theta}{p_{0}+p_{1} / \theta} \mathbb{E}\left[\left|V_{k}(\check{T})\right| \mathbb{I}_{A_{1}^{c} \cap A_{2}^{c}} \theta^{\check{\ell}} \prod_{j=0}^{m-1} \prod_{x \in V_{j}(\check{T})} Z_{m-j-1}^{d_{x}} \mathbb{E}\left[\prod_{j=0}^{m-1} \theta^{-\left|\mathcal{E}_{j}\right|} \mid \check{T}\right]\right] .
\end{aligned}
$$

Applying (2.31) in reverse it follows that

$$
\mathbb{E}_{m}^{(\theta)}\left[\left|\mathcal{E}_{k}\right| \mathbb{I}_{A_{1}^{c} \cap A_{2}^{c}}\right] \leq \frac{d p_{1} / \theta}{p_{0}+p_{1} / \theta} \mathbb{E}_{m}^{(\theta)}\left[\left|V_{k}(\check{T})\right| \mathbb{I}_{A_{1}^{c} \cap A_{2}^{c}}\right]
$$

We bound the last expectation using stochastic domination. Indeed, both $\left|V_{k}(\check{T})\right|$ and $\mathrm{II}_{A_{1}^{c} \cap A_{2}^{c}}$ are increasing functions of $\omega$. Hence from (2.14)

$$
\mathbb{E}_{m}^{(\theta)}\left[\left|V_{k}(\check{T})\right| \mathbb{I}_{A_{1}^{c} \cap A_{2}^{c}}\right] \leq \mathbb{E}^{+}\left[\left|V_{k}(\check{T})\right| \mathbb{I}_{A_{1}^{c} \cap A_{2}^{c}}\right]
$$

Write $p_{i}^{+}=e^{-\beta^{+}}\left(\beta^{+}\right)^{i} / i$ ! for the Poisson probabilities with parameter $\beta^{+}$, and $p_{\geq i}^{+}=$ $p_{i}^{+}+p_{i+1}^{+}+\cdots$. By a recursive computation using independence we see that

$$
\mathbb{E}^{+}\left|V_{k}(\check{T})\right|=\left(d p_{\geq 2}^{+}\right) \mathbb{E}^{+}\left|V_{k-1}(\check{T})\right|=\cdots=\left(d p_{\geq 2}^{+}\right)^{k}
$$

We also have

$$
\left|V_{k}(\check{T})\right| \mathbb{I}_{A_{1}}=\delta_{k, 0} \mathbb{I}_{A_{1}}, \quad\left|V_{k}(\check{T})\right| \mathbb{I}_{A_{2}}=\left(\delta_{k, 0}+\delta_{k, 1}\right) \mathbb{I}_{A_{2}} .
$$


Using (2.29) we find that

$$
\begin{aligned}
\mathbb{P}_{m}^{(\theta)}\left(A_{1}^{c} \cap A_{2}^{c} \cap \Sigma_{m}^{\rho}\right) \leq & \frac{d p_{1} / \theta}{p_{0}+p_{1} / \theta} \sum_{k=0}^{m} \sigma_{m-k-1} \mathbb{E}^{+}\left[\left|V_{k}(\check{T})\right| \mathbb{I}_{A_{1}^{c} \cap A_{2}^{c}}\right] \\
& =\frac{d p_{1} / \theta}{p_{0}+p_{1} / \theta}\left(\sigma_{m-1}\left(1-\mathbb{P}^{+}\left(A_{1} \cup A_{2}\right)\right)+\sigma_{m-2}\left(d p_{\geq 2}^{+}-\mathbb{P}^{+}\left(A_{2}\right)\right)\right. \\
& \left.+\sum_{k=2}^{m} \sigma_{m-k-1}\left(d p_{\geq 2}^{+}\right)^{k}\right) \\
& \leq c_{0}\left(\sigma_{m-1} \frac{c_{1}}{d^{2}}+\sigma_{m-2} \frac{c_{2}}{d^{2}}+\sum_{k=2}^{m} \sigma_{m-k-1}\left(\frac{c_{3}}{d}\right)^{k}\right),
\end{aligned}
$$

for constants $c_{0}, \ldots, c_{3}$ uniform in $d$. Now we use that there is some $c_{4}>0$, uniform in $d$, such that $\sigma_{m-1} \leq c_{4} \sigma_{m}$ for all $m \geq 0$. (This can be seen e.g. by considering the event that $A_{1}$ occurs and that $\left(x, t_{x}\right)$ lies in a loop reaching generation $m$ in its subtree, where $x$ is some fixed child of $\rho$ and $t_{x}$ is the 'time' of the incoming link from $\rho$. This gives $\sigma_{m} \geq \sigma_{m-1} \mathbb{P}_{m}^{(\theta)}\left(A_{1}\right)$.) It follows that

$$
\mathbb{P}_{m}^{(\theta)}\left(A_{1}^{c} \cap A_{2}^{c} \cap \Sigma_{m}^{\rho}\right) \leq \frac{C^{\prime}}{d^{2}}\left(\sigma_{m-1}+\sigma_{m-2}+\sigma_{m-2} \sum_{k=2}^{\infty}\left(\frac{c_{3} c_{4}}{d}\right)^{k-2} .\right) .
$$

The last sum converges if $d$ is large enough, and this establishes Proposition 2.5.

Acknowledgements We thank the anonymous referees for several helpful suggestions to improve the presentation. The research of JEB is supported by Vetenskapsrådet Grant 2015-05195.

Open Access This article is distributed under the terms of the Creative Commons Attribution 4.0 International License (http://creativecommons.org/licenses/by/4.0/), which permits unrestricted use, distribution, and reproduction in any medium, provided you give appropriate credit to the original author(s) and the source, provide a link to the Creative Commons license, and indicate if changes were made.

\section{References}

1. Aizenman, M., Nachtergaele, B.: Geometric aspects of quantum spin states. Commun. Math. Phys. 164, 17-63 (1994)

2. Angel, O.: Random infinite permutations and the cyclic time random walk. Discrete Math. Theor. Comput. Sci. Proc., 9-16 (2003)

3. Barp, A., Barp, E.G., Briol, F.-X., Ueltschi, D.: A numerical study of the 3D random interchange and random loop models. J. Phys. A 48, 345002 (2015)

4. Betz, V., Ehlert, J., Lees, B.: Phase transition for loop representations of quantum spin systems on trees (2018). arXiv: 1804.00860

5. Björnberg, J.E., Ueltschi, D.: Decay of transverse correlations in quantum Heisenberg models. J. Math. Phys. 56, 043303 (2015)

6. Björnberg, J.E., Ueltschi, D.: Critical parameter of random loop model on trees. Ann. Appl. Probab. 28(4), 2063-2082 (2018)

7. Fridman, YuA, Kosmachev, O.A., Klevets, PhN: Spin nematic and orthogonal nematic states in $S=1$ non-Heisenberg magnet. J. Magn. Magn. Mater. 325, 125-129 (2013)

8. Georgii, H.-O., Küneth, T.: Stochastic comparison of point random fields. J. Appl. Probab. 34, 868-881 (1997)

9. Goldschmidt, C., Ueltschi, D., Windridge, P.: Quantum Heisenberg models and their probabilistic representations, in Entropy and the Quantum II. Contemp. Math. 552, 177-224 (2011)

10. Hammond, A.: Infinite cycles in the random stirring model on trees. Bull. Inst. Math. Acad. Sin. 8, 85-104 (2013) 
11. Hammond, A.: Sharp phase transition in the random stirring model on trees. Probab. Theory Relat. Fields 161, 429-448 (2015)

12. Hammond, A., Hegde, M.: Critical point for infinite cycles in a random loop model on trees. arXiv: 1805.11772

13. Sandvik, A.W.: Critical temperature and the transition from quantum to classical order parameter fluctuations in the three-dimensional Heisenberg antiferromagnet. Phys. Rev. Lett. 80, 5196 (1998)

14. Tanaka, K., Tanaka, A., Idokagi, T.: Long-range order in the ground state of the $S=1$ isotropic bilinearbiquadratic exchange Hamiltonian. J. Phys. A 34, 8767-8780 (2001)

15. Tóth, B.: Improved lower bound on the thermodynamic pressure of the spin $1 / 2$ Heisenberg ferromagnet. Lett. Math. Phys. 28, 75-84 (1993)

16. Troyer, M., Alet, F., Wessel, S.: Histogram methods for quantum systems: from reweighting to WangLandau sampling. Braz. J. Phys. 34, 377 (2004)

17. Ueltschi, D.: Random loop representations for quantum spin systems. J. Math. Phys. 54, 083301 (2013) 